\title{
Effective interaction energy of water dimer at room temperature: An experimental and theoretical study
}

\section{AUTHOR(S):}

Nakayama, T; Fukuda, H; Kamikawa, T; Sakamoto, Y; Sugita, A; Kawasaki, M; Amano, T; ... Sakaki, S; Morino, I; Inoue, G

\section{CITATION:}

Nakayama, T ... [et al]. Effective interaction energy of water dimer at room temperature: An experimental and theoretical study. JOURNAL OF CHEMICAL PHYSICS 2007, 127(13): 134302.

ISSUE DATE:

2007-10-07

URL:

http://hdl.handle.net/2433/50143

\section{RIGHT:}

Copyright 2007 American Institute of Physics. This article may be downloaded for personal use only. Any other use requires prior permission of the author and the American Institute of Physics. 


\title{
Effective interaction energy of water dimer at room temperature: An experimental and theoretical study
}

\author{
T. Nakayama, ${ }^{\text {a) }}$ H. Fukuda, T. Kamikawa, Y. Sakamoto, A. Sugita, and M. Kawasaki ${ }^{\text {b) }}$ \\ Laboratory of Photochemical Reaction, Department of Molecular Engineering, Kyoto University, \\ Kyoto 615-8510, Japan \\ T. Amano, H. Sato, and S. Sakaki \\ Laboratory of Molecular Theory for Science and Technology, Department of Molecular Engineering, \\ Kyoto University, Kyoto 615-8510, Japan \\ I. Morino \\ National Institute for Environmental Studies, Tsukuba 305-8506, Japan \\ G. Inoue \\ Graduate School for Environmental Studies, Nagoya University, Nagoya 464-8601, Japan
}

(Received 29 May 2007; accepted 26 July 2007; published online 2 October 2007)

Buffer-gas pressure broadening for the $\nu_{1}+\nu_{3}$ band of $\mathrm{H}_{2} \mathrm{O}$ at $1.34-1.44 \mu \mathrm{m}$ for a variety of buffer gases was investigated at room temperature using continuous-wave cavity ring-down spectroscopy. The effective interaction energy of water dimer under room temperature conditions was evaluated from the pressure broadening coefficients for rare gases using Permenter-Seaver's relation. Monte Carlo simulations were performed using $a b$ initio molecular orbital calculations to evaluate the interaction energies for the water dimer at $300 \mathrm{~K}$. In this theoretical calculation, the orientations of the two water molecules were statistically treated. () 2007 American Institute of Physics.

[DOI: $10.1063 / 1.2773726]$

\section{INTRODUCTION}

Water dimer, $\left(\mathrm{H}_{2} \mathrm{O}\right)_{2}$, has an important role in the radiation budget of the terrestrial atmosphere because of continuum absorptions in the visible, ultraviolet, and thermal infrared region. ${ }^{1,2}$ Hence, it is essential to evaluate an accurate value for the abundance at atmospherically relevant temperatures. Experimental studies on the interaction energy of $\mathrm{H}_{2} \mathrm{O}$ have been performed with two methods, thermal conductivity $^{3}$ and infrared absorption. ${ }^{4-8}$ The dimerization enthalpies determined from thermal conductivity and infrared spectra are for the optimum structure. Theoretical studies have also been reported. ${ }^{9-11}$ Usually, theoretical reports are based on the standard ab initio molecular orbital (MO) calculations and only one optimized configuration with the minimum energy is considered, which corresponds to the "frozen" structure at $0 \mathrm{~K}$. A combination of Monte Carlo (MC) simulations and ab initio $\mathrm{MO}$ calculation enabled us to evaluate the temperature dependence of the interaction energies. Bandyopadhyay et al. ${ }^{9}$ performed multicanonical MC simulations of the water dimer over a wide range of temperatures with the interaction computed by ab initio $\mathrm{MO}$ methods, but the level of their computation, RHF, might not be accurate enough to evaluate the small interaction energy of the water dimer.

In the present work, the effective interaction energy of

\footnotetext{
${ }^{a)}$ Present address: Solar-Terrestrial Environment Laboratory and Graduate School of Science, Nagoya University, Furocho, Chikusa-ku, Nagoya 4648601, Japan.

b) Author to whom correspondence should be addressed. Fax: +81-75-3832573; Electronic mail: kawasaki@moleng.kyoto-u.ac.jp
}

the water dimer at room temperature was evaluated with pressure broadening measurements using Permenter-Seaver's relation. ${ }^{12,13}$ We have measured the pressure broadening coefficients in the $\nu_{1}+\nu_{3}$ band of $\mathrm{H}_{2} \mathrm{O}$ at 1.34-1.44 $\mu \mathrm{m}$ with noble gases as well as $\mathrm{N}_{2}$ and $\mathrm{O}_{2}$ with cavity ring-down spectroscopy (CRDS) using a tunable diode laser. First, the present experimental results were compared with the HITRAN database and the available literature data to check the reliability of our CRDS system. Second, the effective interaction energy at room temperature for $\left(\mathrm{H}_{2} \mathrm{O}\right)_{2}$ has been obtained by applying an energy transfer model to the observed pressure broadening coefficients of $\mathrm{H}_{2} \mathrm{O}$ for noble gases. Finally, we have evaluated the interaction energies by using the MC simulation based on the ab initio MO calculation.

\section{EXPERIMENTAL AND THEORETICAL METHODS}

The experimental setup used in the present study is similar to that descried in our previous studies. ${ }^{14}$ An external cavity diode laser (Santec Co., TSL-210V) was used as the near-infrared light source. The output laser beam, deflected by an acousto-optical modulator (Isomet), was directed into an optical cavity that consisted of two high reflectivity mirrors (II-VI Optics or Kuramoto Co.) with a separation of $60 \mathrm{~cm}$. One of the two mirrors was incorporated in a tubetype piezoactuator (Piezomechanik, HPSt 150/20) for cavity length modulation, with a rate of about $300 \mathrm{~Hz}$. The transmitted light from the cavity was directed to an InGaAs photodiode detector (Hamamatsu Photonics, G5851-11) attached to a preamplifier (NF, SA220F5). When the transmitted beam reached a threshold level, the deflected beam was switched 
off by the acousto-optical modulator crystal and the light stored within the cavity started to ring down.

In the presence of an absorbing species, the light intensity within the cavity is given by the expression

$$
I(t)=I_{0} \exp (-t / \tau)=I_{0} \exp \left(-t / \tau_{0}-\sigma N c t\right),
$$

where $I_{0}$ and $I(t)$ are the light intensities at time 0 and $t, \tau$ is the cavity ring-down time in the presence of an absorbing sample, $\tau_{0}$ is the cavity ring-down time without the absorber present (typically $1.2 \mu \mathrm{s}$ ), and $c$ is the velocity of light. $N$ and $\sigma$ are the concentration and absorption cross section of the absorbing species, respectively. The exponential decay data were digitized with an analog to digital converter (National Instruments, NI5122 $100 \mathrm{MHz}, 14$ bits) and processed using LabVIEW software. Our program averaged the profile of several ring-down wave forms, for which individual ringdown decay curves except for the very initial part of the decay were fitted to a single-exponential function. Sixteen ring-down events were averaged for each spectral data point, thus, a complete scan took about 5-10 min.

Pressure broadening by various buffer gases $\left(\mathrm{N}_{2}, \mathrm{O}_{2}, \mathrm{He}\right.$, $\mathrm{Ne}, \mathrm{Ar}, \mathrm{Kr}$, and $\mathrm{Xe}$; 60-500 Torr) was measured at a fixed partial pressure of $\mathrm{H}_{2} \mathrm{O}$ (0.04 Torr). Three or four measurements of the spectra were performed at each pressure condition. The gases (except $\mathrm{Kr}$ and $\mathrm{Xe}$ ) were flowed with the use of mass-flow controllers (Kofloc, model 3660). Pressure and temperature of the sample gases in the cavity were monitored by pressure gauges (MKS, Baratron 622) and thermocouples, respectively, at the monitor ports. All experiments were performed at $298 \pm 2 \mathrm{~K}$.

We performed the MC simulation combined with the $a b$ initio MO method to evaluate the interaction energies of water dimer. The metropolis algorithm with the standard umbrella sampling technique was used to generate various configurations of water dimer at $300 \mathrm{~K}$. The geometry of the molecule is fixed at the experimental one $\left(R_{\mathrm{OH}}=0.9579\right.$, $\left.\angle \mathrm{HOH}=104 \cdot 5^{\circ}\right) \cdot \mathrm{MP} 2 / 6-31++\mathrm{G}(\mathrm{d}, \mathrm{p})$ level of computation was employed to evaluate the interaction energy at each configuration. The effective interaction energy was then computed as the statistical average over these configurations. Mrazek and Burda ${ }^{15}$ suggested that this level of ab initio MO computations provided reasonable accuracy for the interaction energy. We would like to emphasize that this is a reliable computational level from the standpoint that thousands of configurations are needed to be sampled to evaluate the averaged interaction. Although it is time consuming compared to empirical force field or to Hartree-Fock method, the electron correlation often plays a crucial role to evaluate the accurate interaction energy.

\section{RESULTS AND DISCUSSION}

\section{A. Pressure broadening coefficients for $\mathrm{N}_{2}, \mathrm{O}_{2}$, air, and noble gases: A comparison with the HITRAN database}

The database for the pressure broadening coefficients in the near-infrared region has been accumulated with the use of Fourier transform spectroscopy (FTS) and tunable diode laser spectroscopy (TDLS). To check the reliability of our

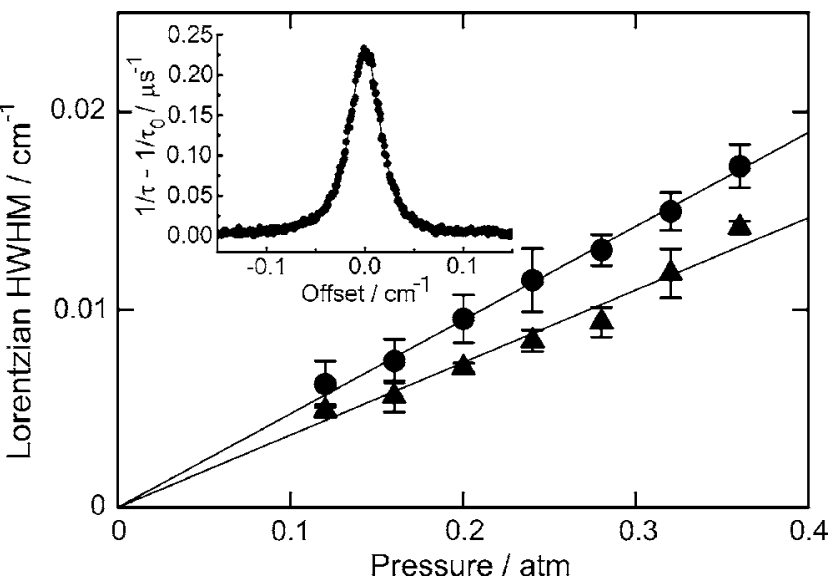

FIG. 1. Lorentzian widths (HWHM) for the $\left(10_{37} \leftarrow 9_{36}\right)$ transition of the $\nu_{1}+\nu_{3}$ band of $\mathrm{H}_{2} \mathrm{O}$ at $298 \mathrm{~K}$ as a function of pressure of buffer gases. Error bars indicate one standard deviation determined by the profile-fit procedure. : Kr, $\boldsymbol{\Delta}$ : Ar. The inset shows an absorption profile for the $\left(10_{37} \leftarrow 9_{36}\right)$ transition of the $\nu_{1}+\nu_{3}$ band of $\mathrm{H}_{2} \mathrm{O}$ broadened for 210 Torr of $\mathrm{Kr}$ at $298 \mathrm{~K}$. Filled circles represent experimental data. The solid curve represents a calculated spectrum with the Voigt function.

CRDS measurements, the air-broadening coefficients were compared with the HITRAN database ${ }^{16}$ and available literature data. ${ }^{17,18}$ An example of the measured profile for the $\left(J_{K_{a}^{\prime} K_{c}^{\prime}}^{\prime}-J_{K_{a}^{\prime \prime} K_{c}^{\prime \prime}}^{\prime \prime}\right)=\left(10_{37}-9_{36}\right)$ transition of the $\nu_{1}+\nu_{3}$ band of $\mathrm{H}_{2} \mathrm{O}$, which was broadened by 210 Torr of $\mathrm{Kr}$, is shown in the inset of Fig. 1, in which the best-fit Voigt profile is shown by the solid curve. The Gaussian component was constrained to the room temperature Doppler width. The data obtained for the half-width at half maximum (HWHM) of the Lorentzian component are plotted as a function of buffer gas pressure. A straight-line fit provides the pressure broadening coefficient $\gamma$ in units of $\mathrm{cm}^{-1} \mathrm{~atm}^{-1}$, as shown in Fig. 1. A small contribution $(<2 \%)$ of self-broadening was taken into account.

The obtained $\gamma$ values of $\mathrm{H}_{2} \mathrm{O}$ for various buffer gases, $\mathrm{N}_{2}, \mathrm{O}_{2}, \mathrm{He}, \mathrm{Ne}, \mathrm{Ar}, \mathrm{Kr}$, and $\mathrm{Xe}$, are listed in Table I. We calculated the air-broadening coefficients $\gamma($ air $)$ using the relation $\gamma($ air $)=0.79 \gamma\left(\mathrm{N}_{2}\right)+0.21 \gamma\left(\mathrm{O}_{2}\right)$, which are listed in Table I and plotted as a function of the rotational quantum number index $(m)$ and quasiquantum number $\left(K_{a}\right)$ in the upper and lower panels in Fig. 2, respectively. The following index, $m$, was used to treat simultaneously the $P$ - and $R$-branch transitions:

$$
\begin{aligned}
& 2 m=J^{\prime}\left(J^{\prime}+1\right)-J^{\prime \prime}\left(J^{\prime \prime}+1\right), \\
& P \operatorname{branch}\left(J^{\prime \prime}-1 \leftarrow J^{\prime \prime}\right), \quad m=-J^{\prime \prime}, \\
& R \operatorname{branch}\left(J^{\prime \prime}+1 \leftarrow J^{\prime \prime}\right), \quad m=J^{\prime \prime}+1 .
\end{aligned}
$$

The data for the transitions of $K_{a}^{\prime}=K_{a}^{\prime \prime}=2, \Delta K_{a}=0$ and $m=9$, $\Delta K_{a}=0$ were plotted in the upper and lower panels in Fig. 2, respectively. The HITRAN database ${ }^{16}$ (solid line) and available literature data reported by Toth $^{17}$ based on FTS (triangles) and Liu et al. ${ }^{18}$ based on TDLS (inverted triangles) are also plotted. Our values are in good agreement with the HITRAN database, ${ }^{16}$ and the literature data reported by Toth $^{17}$ and Liu et al. ${ }^{18}$ 
TABLE I. Buffer-gas pressure broadening coefficients $\gamma$, in the unit of $\mathrm{cm}^{-1} \mathrm{~atm}^{-1}$ for $\mathrm{H}_{2} \mathrm{O}$ at $298 \mathrm{~K}$. (Numbers in parentheses are one standard deviation obtained by the straight-line fitting in units of the last digits quoted.)

\begin{tabular}{|c|c|c|c|c|c|}
\hline $\begin{array}{l}\text { Transition } \\
J_{k_{e}^{\prime} k_{c}^{\prime}}^{\prime}-J_{k_{e}^{\prime \prime} k_{c}^{\prime \prime}}^{\prime \prime}\end{array}$ & & $\mathrm{N}_{2}$ & $\mathrm{O}_{2}$ & $\operatorname{Air}^{\mathrm{b}}$ & \\
\hline $5_{23}-6_{24}$ & & $0.0968(50)$ & $0.0729(60)$ & $0.0918(43)$ & \\
\hline $5_{32}-4_{13}$ & & $0.1034(44)$ & $0.0594(23)$ & $0.0942(35)$ & \\
\hline $9_{27}-8_{26}$ & & $0.0774(34)$ & $0.0411(23)$ & $0.0698(27)$ & \\
\hline $10_{19}-9_{18}$ & & $0.0450(27)$ & $0.0236(27)$ & $0.0405(22)$ & \\
\hline $10_{28}-9_{27}$ & & $0.0784(37)$ & $0.0391(17)$ & $0.0702(29)$ & \\
\hline $10_{37}-9_{36}$ & & $0.0988(30)$ & $0.0503(28)$ & $0.0886(25)$ & \\
\hline $10_{46^{-}}-9_{45}$ & & $0.0854(29)$ & $0.0390(34)$ & $0.0757(24)$ & \\
\hline $10_{55^{-}} 9_{54}$ & & $0.0750(24)$ & $0.301(23)$ & $0.0656(20)$ & \\
\hline $11_{29}-10_{28}$ & & $0.0662(30)$ & $0.0293(16)$ & $0.0585(24)$ & \\
\hline $12_{210^{-}}-11_{29}$ & & $0.0606(43)$ & $0.0217(29)$ & $0.0524(34)$ & \\
\hline $\begin{array}{l}\text { Transition } \\
J_{k_{e}^{\prime} k_{c}^{\prime}-J_{k_{e}^{\prime \prime} k_{c}^{\prime \prime}}^{\prime \prime}}\end{array}$ & $\mathrm{He}$ & $\mathrm{Ne}$ & $\mathrm{Ar}$ & $\mathrm{Kr}$ & $\mathrm{Xe}$ \\
\hline $5_{23}-4_{04}$ & $0.0217(17)$ & $0.0230(13)$ & $0.0420(18)$ & $0.0522(21)$ & $0.0618(25)$ \\
\hline $10_{37}-9_{36}$ & $0.0283(12)$ & $0.0219(27)$ & $0.0356(13)$ & $0.0465(32)$ & $0.0694(30)$ \\
\hline $10_{55^{-}} 9_{54}$ & $0.0135(31)$ & $0.0142(11)$ & $0.0242(22)$ & $0.0358(27)$ & $0.0522(20)$ \\
\hline
\end{tabular}

$\overline{\bar{a}}$ The rotational quantum numbers are in standard asymmetric rotor notation. ${ }^{\mathrm{b}}$ Calculated from $\gamma($ air $)=0.79 \gamma\left(\mathrm{N}_{2}\right)+0.21 \gamma\left(\mathrm{O}_{2}\right)$.

\section{B. Experimental determination of the effective interaction energy of water dimer at $298 \mathrm{~K}$}

The effective interaction energy of $\left(\mathrm{H}_{2} \mathrm{O}\right)_{2}$ was evaluated using the observed broadening coefficients for noble gases. Broadening coefficients for noble gases for the $\left(5_{23} \leftarrow 4_{04}\right)$,
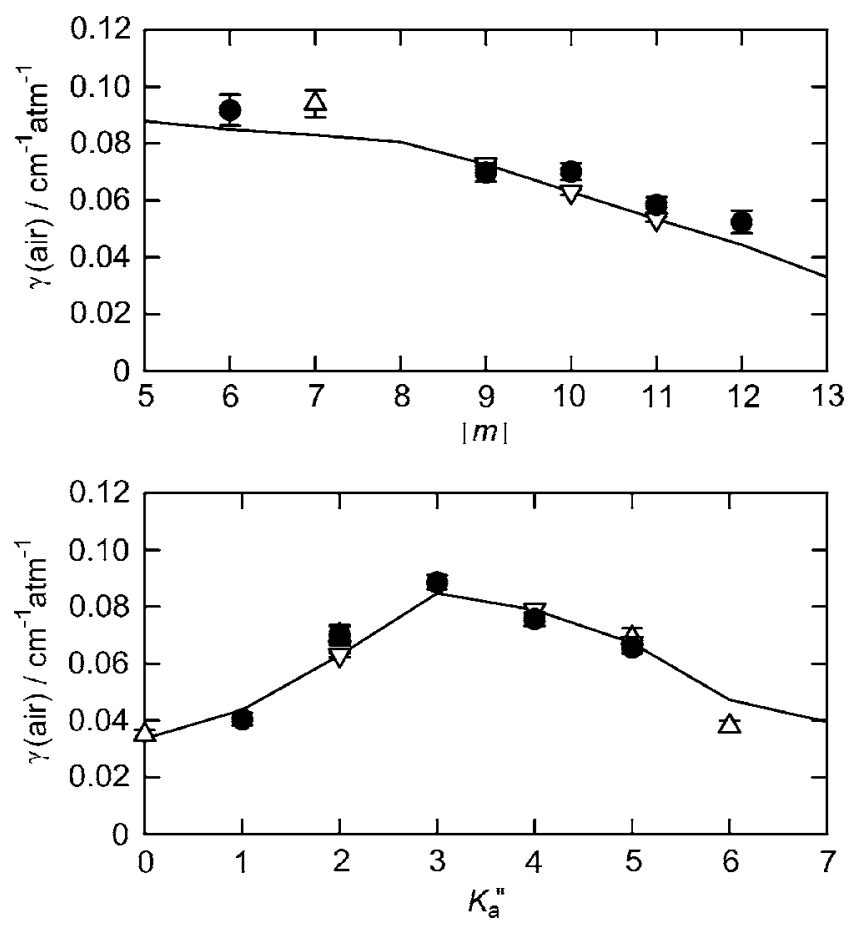

FIG. 2. Pressure broadening coefficients of $\mathrm{H}_{2} \mathrm{O}$ for air as a function of (upper panel) rotational level $|m|$ for $K_{a}^{\prime}=K_{a}^{\prime \prime}=2$, and (lower panel) quasiquantum number $K_{a}^{\prime \prime}$ for $\Delta K_{a}=0$ with $m=9$. ๑: This work, $\nabla$ : Toth (Ref. 17), $\triangle$ : Liu et al. (Ref. 18), solid line: HITRAN (Ref. 16). Error bars indicate one standard deviation determined by the profile-fit procedure.

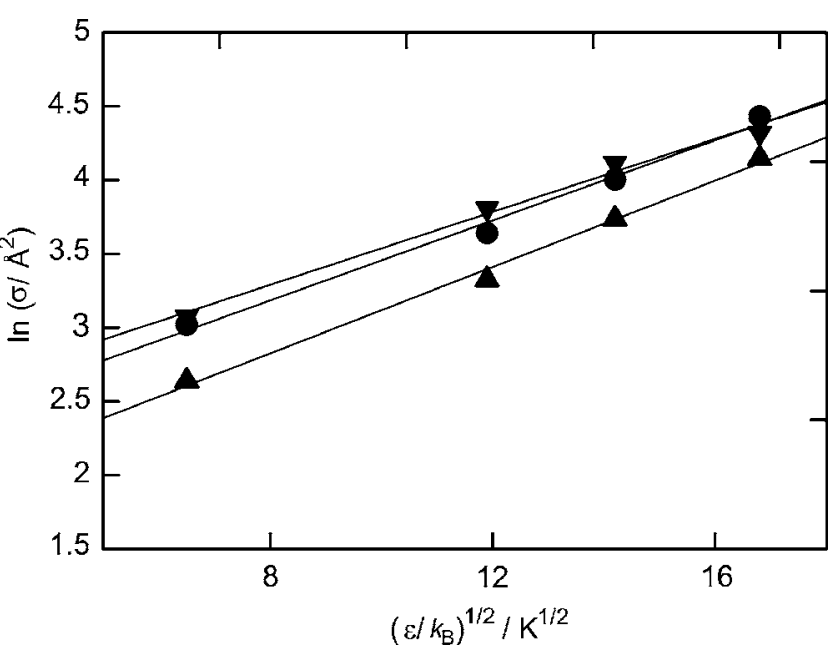

FIG. 3. Plots of the logarithm of the cross section $\sigma_{M}$ for the broadening process of the $\left(5_{23} \leftarrow 4_{04}\right)$ (inverted triangles), $\left(10_{37} \leftarrow 9_{36}\right)$ (circles), and $\left(10_{55} \leftarrow 9_{54}\right)$ (triangles) transitions of the $\nu_{1}+\nu_{3}$ band vs the well depth $\varepsilon_{M M}$ of the dimer of a variety of noble gases (filled circles: $\mathrm{Ne}, \mathrm{Ar}, \mathrm{Kr}$, and $\mathrm{Xe}$ ). Solid lines show the results of a linear least-squares fit of the experimental data. See Eq. (3).

$\left(10_{37} \leftarrow 9_{36}\right)$, and $\left(10_{55} \leftarrow 9_{54}\right)$ transitions of the $\nu_{1}+\nu_{3}$ band were used for the evaluation. Permenter and Seaver ${ }^{19,20}$ have developed a successful model that correlates Boltzmann averaged cross sections $\sigma_{M}$ of a variety of energy transfer processes for a variety of collision partners $M$, with the intermolecular well depths $\varepsilon_{A M}$, for the interaction between the molecule $A$ and a collision partner $M$.

$$
\sigma_{M}=C \exp \left(\varepsilon_{A M} / k_{B} T\right),
$$

where $C$ is a constant, $k_{B}$ is Boltzmann's constant, and $T$ is temperature. The correlation is particularly useful for energy transfer processes which depend upon long range attractive forces. In the Lorentz-Berthelot geometric mean approximation, $\varepsilon_{A M}=\left(\varepsilon_{A A} \varepsilon_{M M}\right)^{1 / 2}$, the equation is conveniently expressed in terms of the well depth $\varepsilon_{A A}$ for the dimer of $A$ and $\varepsilon_{M M}$ for the dimer of $M$,

$$
\ln \left(\sigma_{M}\right)=\ln C+\left(\varepsilon_{A A} / k_{B} T^{2}\right)^{1 / 2} \times\left(\varepsilon_{M M} / k_{B}\right)^{1 / 2} .
$$

Figure 3 shows the Permenter-Seaver plots for the pressure broadening of the $\left.\left(5_{23} \leftarrow 4_{04}\right)\right),\left(10_{37} \leftarrow 9_{36}\right)$, and $\left(10_{55}\right.$ $\left.\leftarrow 9_{54}\right)$ transitions of the $\nu_{1}+\nu_{3}$ band for a variety of colliding rare gases $\left(\varepsilon_{\mathrm{NeNe}} / k_{B}=42.31 \mathrm{~K}, \varepsilon_{\mathrm{ArAr}} / k_{B}=143.33 \mathrm{~K}\right.$, $\varepsilon_{\mathrm{KrKr}} / k_{B}=201.43 \mathrm{~K}$, and $\left.\varepsilon_{\mathrm{XeXe}} / k_{B}=282.88 \mathrm{~K}\right)$. These $\varepsilon_{M M} / k_{B}$ data are taken from the best estimated values compiled by Tang and Toennies. ${ }^{21}$ In Fig. 3, $\sigma_{M}$ is the cross section for the broadening process calculated by converting $\gamma$ to SI units and multiplying by the factor $2 \pi k_{B} T /\left\langle v_{r}\right\rangle$. As shown in Fig. 3, a linear correlation is observed for $\mathrm{Ne}, \mathrm{Ar}$, $\mathrm{Kr}$, and Xe. Linear least-squares fit analysis of the data in Fig. 3 yielded the effective intermolecular well depth (interaction energy at room temperature) for $\left(\mathrm{H}_{2} \mathrm{O}\right)_{2}$ to be $\varepsilon_{\mathrm{H}_{2} \mathrm{OH}_{2} \mathrm{O}} / k_{B}=1360 \pm 200,1630 \pm 180$, and $1900 \pm 210 \mathrm{~K}$ for $\left(5_{23} \leftarrow 4_{04}\right),\left(10_{37} \leftarrow 9_{36}\right)$, and $\left(10_{55} \leftarrow 9_{54}\right)$ transitions, respectively. We chose to cite a final value, the average of the individual determinations together with error limits, which encompass the extremes of the determinations, hence, $\varepsilon_{\mathrm{H}_{2} \mathrm{OH}_{2} \mathrm{O}} / k_{B}=1630 \pm 480 \mathrm{~K}\left(3.24 \pm 0.95 \mathrm{kcal} \mathrm{mol}^{-1}\right)$. 
TABLE II. Dimerization enthalpies and effective interaction energies of $\mathrm{H}_{2} \mathrm{O}$ dimer.

\begin{tabular}{lll}
\hline \hline Method & $\begin{array}{l}\varepsilon_{\mathrm{H}_{2} \mathrm{O}-\mathrm{H}_{2} \mathrm{O}} \\
\left(\mathrm{kcal} \mathrm{mol}^{-1}\right)\end{array}$ & Reference \\
\hline $\begin{array}{ll}\text { (Experimental) } \\
\text { Thermal conductivity }\end{array}$ & $3.59 \pm 0.50^{\mathrm{a}}$ & 3 \\
Infrared absorption & $5.20 \pm 1.50^{\mathrm{a}}$ & 4 \\
Infrared absorption & $4.55^{\mathrm{a}}$ & 5 \\
Infrared absorption & $3.98 \pm 0.90^{\mathrm{a}}$ & 6 \\
Infrared absorption & $3.6 \pm 0.7^{\mathrm{a}}$ & 7 \\
Infrared absorption & $3.80 \pm 0.07^{\mathrm{a}}$ & 8 \\
Pressure broadening & $3.24 \pm 0.95^{\mathrm{b}}$ & This work \\
(Theoretical) & & \\
MC simulation & & \\
\hline \hline
\end{tabular}

${ }^{\mathrm{a}}$ Previous reported values of dimerization enthalpy.

${ }^{\mathrm{b}}$ Effective interaction energy for $298 \pm 2 \mathrm{~K}$.

${ }^{c}$ Monte Carlo simulation combined with the ab initio molecular orbital method. An evaluated zero point energy of $1.22 \mathrm{kcal} \mathrm{mol}^{-1}$ was taken into account (see text).

${ }^{\mathrm{d}}$ Effective interaction energy for $300 \mathrm{~K}$.

The obtained experimental result was listed in Table II with the selected literature experimental data of the dimerization enthalpy of $\mathrm{H}_{2} \mathrm{O}$. The present results provide the effective interaction energy of $\mathrm{H}_{2} \mathrm{O}$ dimer at room temperature. Our experimental value, $3.24 \pm 0.95 \mathrm{kcal} \mathrm{mol}^{-1}$, is smaller by $0.35-1.96 \mathrm{kcal} \mathrm{mol}^{-1}$ than the recent experimental data. This difference could be attributed to the fact that the reported dimerization enthalpy was obtained for the minimum energy geometry in the thermal conductivity and infrared absorption measurements, while our experimental result was obtained for an average over the different orientations of the two colliding water molecules at $298 \mathrm{~K}$, an atmospheric relevant temperature.

\section{Theoretical calculations of the effective interaction energy of water dimer at $300 \mathrm{~K}$}

The integration over various configurations using molecular simulation technique such as MC is necessary to compare with the experimental estimation. Figure 4 shows the distribution of the interaction energy at $300 \mathrm{~K}$. The distribution is typical in the canonical distribution and shows qualitative agreement with that by Bandyopadhyay et al., although they did not show the absolute value. Note that the levels of ab initio computations are different between their simulation and ours, and their energy using RHF/6-31G* slightly underestimates the binding energy. It is noteworthy that the depth shows significant temperature dependence, ${ }^{22}$ that is, the distribution of the interaction energy is shifted to positively greater (shallower) direction with increasing temperature due to the thermal energy in the vibrational modes. The averaged interaction energy from our simulation is $-4.54 \mathrm{kcal} \mathrm{mol}^{-1}$, in which the zero point energy (ZPE) is neglected. The ZPE of the geometry-optimized dimer is $1.22 \mathrm{kcal} \mathrm{mol}^{-1}$. Since ZPEs cannot be evaluated at arbitrary configurations except for the minimum, we estimate the effective interaction energy with the ZPE correction by simply adding the aforementioned ZPE. Our best theoretical estima-

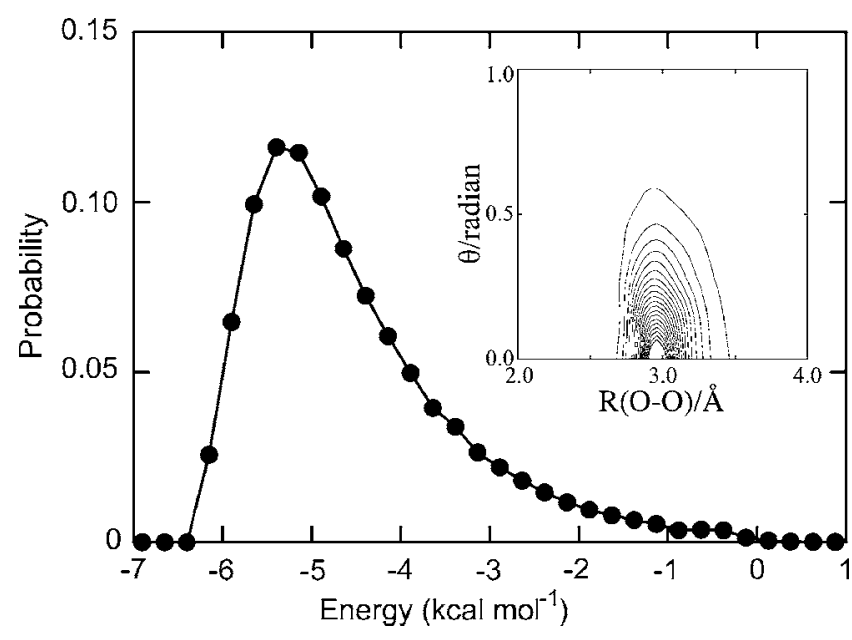

FIG. 4. The distribution of the interaction energies at $300 \mathrm{~K}$ determined by Monte Carlo simulation using ab initio molecular orbital calculation for various configurations of the two $\mathrm{H}_{2} \mathrm{O}$ molecules. The insert shows the distribution function of dimer configurations for the $\mathrm{O}-\mathrm{O}$ distances and the angles of the $\mathrm{H}$ (hydrogen bonding)-O-O.

tion of the effective interaction energy at $300 \mathrm{~K}$ is, thus, $3.32 \mathrm{kcal} \mathrm{mol}^{-1}$. This value agrees well with the experimental one within the margin of the experimental error. It should be noted, however, that a few sources of errors still remain that are difficult to be estimated. One is the basis set superposition error (BSSE) and the other is the contribution from the geometrical relaxation of the water molecule. The former overestimates the interaction while the latter underestimates it. Xantheas et $\mathrm{al}^{23}$ reported that BSSE of the water dimer is $0.83 \mathrm{kcal} \mathrm{mol}^{-1}$ (MP2/aug-cc-pVDZ) at the optimized configuration including the deformation of the molecules. This could be regarded as the maximum limit of the error since the value must be averaged over the various configuration.

\section{ACKNOWLEDGMENTS}

This work is financially supported in part by the Global Environment Research Fund (project B-2) of Ministry of the Environment of Japan, and Grant-in Aid for Scientific Research on Priority Areas "Water and biomolecules" (43018031019) and "Molecular Theory for Real Systems" (461) of Ministry of Education, Culture, Sports, Science and Technology of Japan.

\footnotetext{
${ }^{1}$ K. Pfeilsticker, A. Lotter, C. Peters, and H. Bosch, Science 300, 2078 (2003).

${ }^{2}$ B. Sierk, S. Solomon, J. S. Daniel, R. W. Portmann, S. I. Gutman, A. O. Langford, C. S. Eubank, E. G. Dutton, and K. H. Holub, J. Geophys. Res. 109, D08307 (2004).

${ }^{3}$ L. A. Curtiss, D. J. Frurip, and M. Blander, J. Chem. Phys. 71, 2703 (1979).

${ }^{4}$ H. A. Gebbie, W. J. Burroughs, J. Chamberlain, J. E. Harries, and R. G. Jones, Nature (London) 221, 143 (1969).

${ }^{5}$ V. I. Dianov-Klokov, V. M. Ivanov, V. N. Arefev, and N. I. Sizov, J. Quant. Spectrosc. Radiat. Transf. 25, 83 (1981).

${ }^{6}$ G. V. Bondarenko and Y. E. Gorbaty, Mol. Phys. 74, 639 (1991).

${ }^{7}$ Y. S. Jin and S. Ikawa, J. Chem. Phys. 119, 12432 (2003).

${ }^{8}$ J. G. Cormier, J. T. Hodges, and J. R. Drummond, J. Chem. Phys. 122, 114309 (2005).

${ }^{9}$ P. Bandyopadhyay, S. Ten-no, and S. Iwata, Mol. Phys. 96, 349 (1999).

${ }^{10}$ R. S. Fellers, C. Leforestier, L. B. Barly, M. G. Brown, and R. J. Saykally, Science 284, 945 (1999).

${ }^{11}$ E. M. Mas, R. Bukowski, K. Szalewicz, G. C. Groenenboom, P. E. S.
} 
Wormer, and A. van der Avoird, J. Chem. Phys. 113, 6687 (2000).

${ }^{12}$ H. R. Barry, L. Corner, G. Hancock, R. Peverall, T. L. Ranson, and G. A. D. Ritchie, Phys. Chem. Chem. Phys. 5, 3106 (2003).

${ }^{13}$ T. Nakayama, H. Fukuda, A. Sugita, S. Hashimoto, M. Kawasaki, S. Aloisio, I. Morino, and G. Inoue, Chem. Phys. 334, 196 (2007).

${ }^{14}$ S. Nakamichi, Y. Kawaguchi, H. Fukuda, S. Enami, S. Hashimoto, M. Kawasaki, T. Umekawa, I. Morino, H. Suto, and G. Inoue, Phys. Chem. Chem. Phys. 8, 364 (2006).

${ }^{15}$ J. Mrazek and J. V. Burda, J. Chem. Phys. 125, 194518 (2006).

${ }^{16}$ L. S. Rothman et al., J. Quant. Spectrosc. Radiat. Transf. 96, 139 (2005).

${ }^{17}$ R. A. Toth, J. Quant. Spectrosc. Radiat. Transf. 94, 1 (2005).

${ }^{18}$ X. Liu, X. Zhou, J. B. Jeffries, and R. K. Hanson, J. Quant. Spectrosc.
Radiat. Transf. 103, 565 (2007).

${ }^{19}$ H.-M. Lin, M. Seaver, K. Y. Tang, A. E. W. Knight, and C. S. Parmenter, J. Chem. Phys. 70, 5442 (1979).

${ }^{20}$ C. S. Parmenter and M. Seaver, J. Chem. Phys. 70, 5458 (1979).

${ }^{21}$ K. T. Tang and J. P. Toennies, J. Chem. Phys. 118, 4976 (2003).

${ }^{22}$ The Monte Carlo simulations show evident temperature dependency. The averaged interaction energies evaluated with a lower level of $a b$ initio molecular method [Hartree-Fock $/ 6-31 \mathrm{G}(\mathrm{d}, \mathrm{p})]$ are $-5.2 \mathrm{kcal} / \mathrm{mol}(50 \mathrm{~K})$, $-4.8 \mathrm{kcal} / \mathrm{mol}(100 \mathrm{~K}),-4.5 \mathrm{kcal} / \mathrm{mol}(150 \mathrm{~K})$, and $-3.9 \mathrm{kcal} / \mathrm{mol}$ (200 K).

${ }^{23}$ S. S. Xantheas, C. J. Burnham, and R. J. Harrison, J. Chem. Phys. 116, 1493 (2002) 